\title{
Measuring the Role of Way of Thinking in Life and Medicine
}

\author{
Dr. Maria Kuman* \\ Holistic Research Institute, USA \\ *Corresponding author: Maria Kuman PhD, Holistic Research Institute, 1414 Barcelona, Knoxville, TN 37923, USA; Email: holisticare1@gmail.com
}

Submission: 眥 April 09, 2018; Published: 眥 May 29, 2018

\begin{abstract}
The lifespan of medical doctors is in average 58.5 years, while the lifespan of the ordinary people is 75 years. This article offers explanation based on measurements of the human nonlinear electromagnetic field (NEMF) that this is so because our doctors deal with sick people all the time. The only way to help the doctors live the lifespan of ordinary people is to switch our medicine from healing diseases and dealing with sick people to preventing diseases and dealing with healthy people. The doctors will be paid even more than they are paid now because everybody will have a doctor and will be visiting his doctor for regular checkups four times a year (This is the ancient Chinese system-read the article for details). For this to happen, our doctors must know our body as a whole. There will be no: cardiologist, who deals only with diseases of the heart, gastroenterologists, etc. Also to be able to practice the medicine of the $21^{\text {st }}$ century and predict and prevent diseases, the doctors need not only to know the whole body, they need to become familiar with the human NEMF, which we measure, and acknowledge the role emotions play for our health and wellbeing. Then our medicine will become Info Energy Medicine.
\end{abstract}

Keywords: Positive thinking and health; Negative thinking and disease; Positive emotions and health; Negative emotions and disease; Necessity of preventive medicine

\section{Introduction}

\section{Measuring the effect of negative thinking-its role in the onset of chronic diseases and cancer}

In a number of articles [1-3] and books [4,5], I have emphasized the role of stress in the onset of chronic diseases and cancer. We are living in the $21^{\text {st }}$ century and it is high time to start considering the fact that we are emotional creatures and not just a bag of chemical substances that react with each other in a specific way.

To determine the role the way of thinking and emotions play in our life and health, I did a series of measurements with my patented sensitive energy meter. I asked people to think about the happiest moment of their life and I measured their energy. Then I asked these people to think about the saddest moment in their life and I measured them again.

The results are presented on Figure 1. They show that when thinking about the happiest moment of their life, the energy was high and the body was much better balanced. Straight horizontal line means perfect health. Therefore, when we feel happy, we are healthy. When thinking about the saddest moment of their life, the whole energy dropped down and became more unbalanced. Therefore, each sad thought makes us less healthy. They are measured at the body energy centers (horizontal axis), which are alternating vortices and anti-vortices of the human nonlinear electromagnetic field (NEMF), which has the shape of a torus (Figure 2).

Hans Selye is the Godfather of stress. He borrowed the word "stress" from engineering and first applied it to psychological stress. Then he devoted 40 years of his life studding stress. He found that the same stress causes different diseases in different individuals because just as a material under stress would collapse at the weakest place, under psychological stress the genetically inherited weak organ collapses first.

Our measurements found that one negative thought was enough for the genetically inherited weak organ to drop in energy. This proves that not only does each negative emotion shift the genetically inherited organ a step farther to a chronic disease of the organ, each negative thought do the same.

\section{Dynamic of our NEMF revealed through two independent sets of measurements}

Then we did another set of measurements with our patented sensitive energy meter on energy healers, called Reiki Healers, who heal with hands. The results are published in [6]. Rei-Ki (Chi) means Universal energy and they claim they use Universal energy to heal. Our measurement proved that they indeed suck Universal (electrical) energy from the atmosphere. We found that the energy 
enters their body through the top of their head because after each treatment the energy on top of their heads was higher [6].

The Russian scientist Shkatov found with his patented torsemeter [7] that positive emotions make our torus shaped nonlinear electromagnetic field (NEMF) (Figure 2) to spin faster clockwise, while negative emotions make our NEMF spin faster counterclockwise. When we compare our measurements with the measurements of Shkatov, the following dynamic picture emerges.

Our measurements showed that at positive thinking the energy of the body increases. Another set of measurements of Reiki healers showed that their energy on top of the head increases after each energy treatment, which means that the energy is sucked through the top of the head. Let us combine this with tee measurement of Shkatov, which showed that when we experience positive emotions (in our case positive thinking) our NEMF spin faster clockwise.

Vortices spin clockwise and suck energy. Here is the whole picture - when the torus spins clockwise like a vortex, energy is sucked through the hole of the donut (Figure 2). When we experience negative emotions (or just at negative thinking) our NEMF spin faster counterclockwise, like an anti-vortex, and energy is emitted through the hole of the donut, which explains the drop of energy at negative (sad) thinking (see Figure 1).

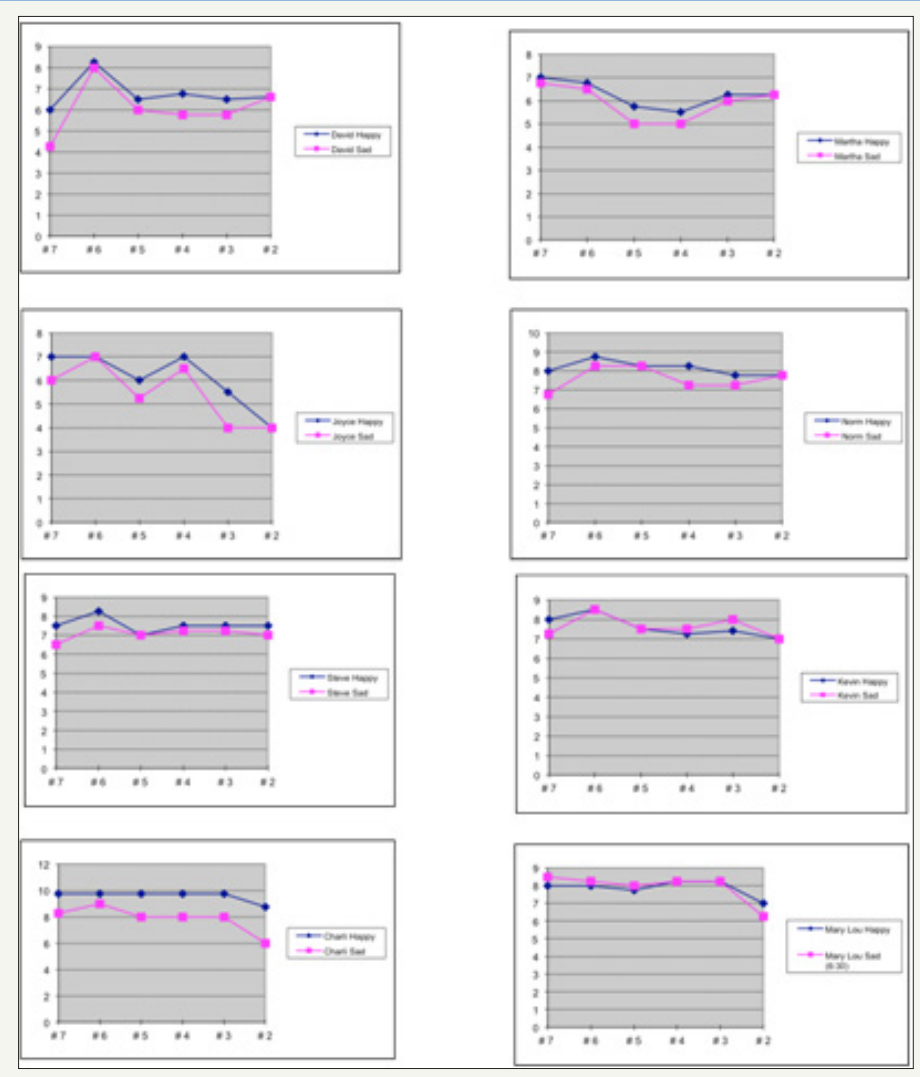

Figure 1: On the vertical axis are the measured electric currents in microamperes when different individuals think about the happiest moment of their life (upper curve) and when they think about the saddest moment of their life (lower curve).

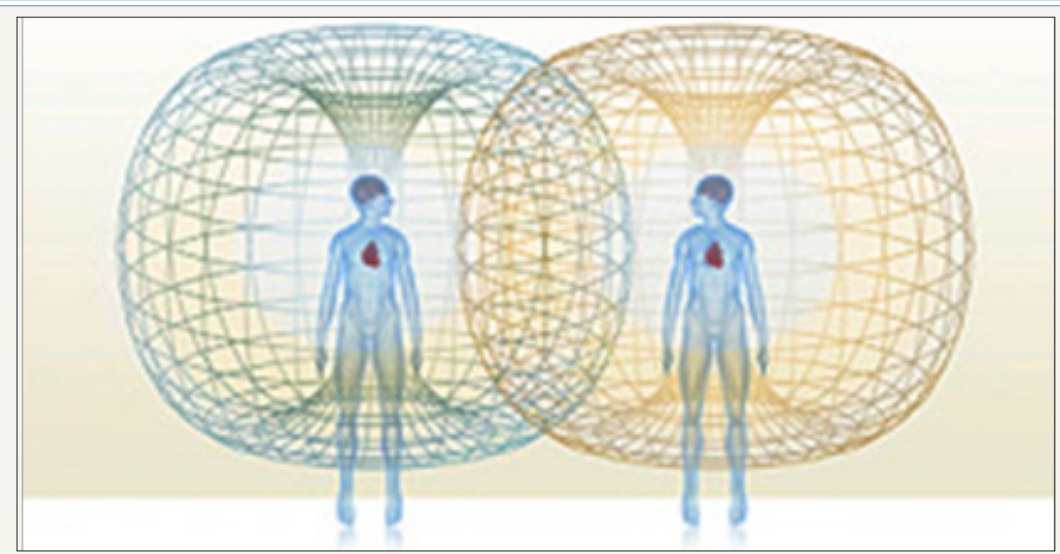

Figure 2: The torus-shaped NEMF of humans. 
In the article [8], we underlined that cancer results from negative emotions, negative thinking, or negative stress because they gobble the energy of the person's NEMF and this NEMF is the field that rules and controls everything in the body including the control on cell division. When this NEMF is very weak and there is nothing to control the cellular growth, the cells start to divide out of control and this is the beginning of the malignancy called cancer.

The sensitivity of our NEMF to sound and light opens totally new info-energetic ways to counter-act cancer [9]. Details will be given in another article.

\section{Two stars have the same donut shape NEMF with the same dynamics as two men}

When I compared our measurements with these of Shkatov [7], I saw the global picture. When the torus spins clockwise (at positive emotions or positive thinking), energy is sucked through the hole of the donut, just like a vortex spinning clockwise would do. When the torus spins counterclockwise (at negative emotions or negative thinking), energy is released through the hole of the donut, just like an anti-vortex spinning counterclockwise would do [10].

I was amazed because in astronomy, similar dynamic was observed in double stars, which have the same donut shape NEMF. The dimmer star through the hole of its donut would suck energy from the donut's hole of the brighter star. This continues until the energy of both stars becomes equal $[11,12]$.

I was seeing in my mind the dynamic of this star interaction The dimmer star, while spinning faster and faster clockwise, was sucking energy through the hole of its donut from the brighter star and shining brighter and brighter. The brighter star, while spinning counterclockwise, was losing energy through the hole of its donut and becoming with time dimmer and dimmer.

I couldn't help it but think about our doctors dealing with sick people - sick because of dominant negative thinking and negative emotions. The donut shaped NEMF of these people would be spinning counterclockwise and sucking energy through the hole of the donut on top of their heads (Figure 2) (just like the dimmer stars), while the doctors would be losing energy through the hole of their donut shaped NEMF on top of their heads (Figure 2) like the brighter stars.

I think this could explain why the doctors live in average only 58.5 years while the rest of the population lives 75 years. If the doctors want to live as long as the rest of the people, they need to switch to practicing preventive medicine and deal with healthy people instead of dealing with sick people. And we need to educate all people about the harm of negative thinking and negative emotions.

\section{Conclusion}

Ancient Chinese wisdom says: "Only the bad doctor cure, the good doctor knows how to prevent the disease." In ancient China, everybody was going to his doctor acupuncturist for check up four times a year, and paying an annual fixed fee. If a deviation from norm would appear, the doctor would eliminate it with appropriate acupuncture treatment and the person would stay healthy.

If a disease would appear, this meant that the doctor didn't do his preventive treatments right. Then to cure the already onset disease, the doctor was supposed to do for free the 14 daily acupuncture treatments necessary to cure the disease. Thus, it was in the interest of the doctor to keep his clients healthy.

If this practice would be accepted today, the doctors would be seeing more people and making more money, but they would be dealing with healthy, not sick people. Then the doctors would live 75 years, just like everybody else, because they would be dealing with healthy individuals. Now they are living only 58.5 years because they deal all the time with sick people.

Why is our medicine the way it is? The Greek philosopher Heraclitus (540 - $480 \mathrm{BC}$ ) intuitively perceived that our Universe is in constant movement and evolution and so is our body, which is constantly changing and unfortunately aging. He was famous with his statement: "Everything changes. You cannot bathe in the same water of the same river twice" [10].

100 years after the death of Heraclitus, the Greek philosopher Aristotle was born (384-322 BC). He was scholar of Plato, but after the death of Plato he switched to empiricism. He started teaching that we can understand everything by studding it piece by piece regardless was it universe or human body [10].

Unfortunately, the Aristotle's mechanistic view prevailed and still rules in the $21^{\text {st }}$ century 2,300 years later. Isn't it time to stop this nonsense? We developed précised instruments, like the Hubble's and other telescopes, which allow us to see what is going on in the Universe-seeing we see, but we do not understand what is going on. Neither can we comprehend how our Universe was created and functions [10].

Just as pitiful is the situation with understanding how our body functions and how to fix it when it malfunctions. We use the Aristotle's mechanistic approach. The body is divided into parts and the doctors study or specialize only in one part: cardiologist, gastroenterologist, etc. If so, we cannot expect our narrowspecialized doctors to know what is causing our symptoms because the cause could be in area he is not specialized in [12].

The techniques used to treat health problems are based on the same mechanistic approach. The contemporary surgeon is like "bio Plummer", who cuts off the part that is worn out or clogged and replace it with metal or plastic one that works. Just like in pluming or car repair.

The surgeons replace warned knee joints with metal ones, they clean the congested arteries in the way the congested water pipes are cleaned. First, they use chemicals to dissolve the plagues. When this does not work, they push mechanically the plagues with a piston. The third approach is "bypass" - they use a plastic tube to bypass the congested area [12]. 
The present doctors would have difficulties overcoming the psychological barrier of their narrow specializations to become whole body doctors. However, to be good doctors they need to know how the body functions as a whole. In other words, they need to know and practice integrated medicine.

Should the dynamic concept of the Greek philosopher Heraclitus stay, we would have known long time ago the dynamic of creation and functioning of our Universe and the dynamic of functioning of our body. We would also know the dynamic of each disease we suffer, which would allow us to prevent these diseases instead of being victims of them [10].

\section{References}

1. Kuman M (2018) International Journal of Integrated Medicine.

2. Kuman M (2018) Advances in Complimentary and Alternative Medicine $1(3)$.
3. Kuman M (2018) International Journal of Complementary and Alternative Medicine (1).

4. Kuman M (1993) What everybody have to know about chronic pain, chronic diseases, and cancer. Health and Happiness Books.

5. Kuman M (1997) Modern aspects of ancient acupuncture. Health and Happiness Books.

6. Kuman M (2017) International Journal of Integrated Medicine 42(3-4).

7. Tihoplav V, Tihoplav T, Miroznanie (2012) Saint Petersburg, Russia.

8. Kuman M (2018) Advances in Complimentary and Alternative Medicine $1(4)$.

9. Burgay M (2004) Nature 426: 531-533.

10. Kuman M (2017) The same fields and dynamics in stars and man. Health and Happiness Books.

11. Lyne AG (2004) Science.

12. Tihoplav V, Tihoplav T (2003) Garmonia haosa (The Harmony of the Chaos), Russia.
Creative Commons Attribution 4.0

International License

For possible submissions Click Here

\section{Submit Article}

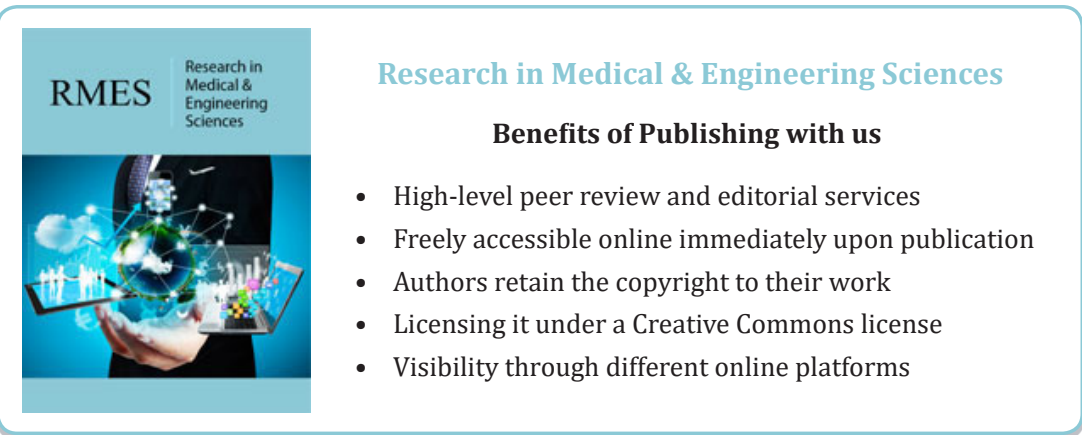

